\title{
Synthesis and Studies on Anticonvulsant and Antidepressant Activities of 5-Alkoxy-tetrazolo[1,5-a]quinolines
}

\author{
Xian-Qing Deng, ${ }^{\ddagger}$ Cheng-Xi Wei, ${ }^{\dagger \neq}$ Ming-Xia Song, ${ }^{\ddagger}$ Kyu-Yun Chai, ${ }^{\S}$ Zhi-Gang Sun, ${ }^{\dagger, *}$ and Zhe-Shan Quan ${ }^{\ddagger}$ \\ ${ }^{\dagger}$ Institute of Neurosurgery, Inner Mongolia University for Nationalities, No. 1742, Holin River Street, \\ Tongliao, Inner Mongolia Autonomous Region 028007, China. *E-mail: sunzhigang101@yahoo.cn \\ `College of Pharmacy, Yanbian University, No. 1829, JuZi Street, Yanji, Jilin 133000, China. *E-mail: zsquan@ybu.edu.cn \\ ${ }^{\S}$ Department of chemistry, Wonkwang University, Iksan, 570-749, Korea \\ Received November 13, 2009, Accepted January 4, 2010
}

\begin{abstract}
A series of 5-alkoxy-tetrazolo[1,5-a]quinolines were synthesized to evaluate their anticonvulsant and antidepressant effects. Anticonvulsant effects and neurotoxicity of the compounds when injected intraperitoneally to mice were determined by a maximal electroshock (MES) test and a rotarod test, respectively. Only three of the synthesized compounds $(\mathbf{4 a}, \mathbf{4 b}, \mathbf{4 c})$ displayed anticonvulsant activity at a dose of $300 \mathrm{mg} / \mathrm{kg}$. Most of the compounds significantly reduced immobility times during the forced swimming test (FST) at a dose of $100 \mathrm{mg} / \mathrm{kg}$, indicative of antidepressant activity. Among the compounds, 5-(2-fluorobenzyloxy)tetrazolo[1,5-a]quinoline (4k) reduced immobility time by $66.85 \%$ at $30 \mathrm{mg} / \mathrm{kg}$ compared with the same dose of Fluoxetine, which reduced immobility time by $52.30 \%$. According to the results of the 5-Hydroxytryptophan induced head-twitch test and yohimbine toxicity potentiation test, the noradrenergic system seems not to be involved in the antidepressant-like effect of compound $4 \mathrm{k}$ while the serotonergic system seems a little to be involved.
\end{abstract}

Key Words: Synthesis, Tetrazolo[1,5-a]quinoline, Anticonvulsant, Antidepressant, Forced swimming test

\section{Introduction}

Increasing evidence suggests that quinolinone derivatives possess a broad spectrum of biological activities such as antitumor, ${ }^{1}$ antimalarial, ${ }^{2}$ antiplatelet, ${ }^{3}$ antidepressant, ${ }^{4}$ antiulcer, ${ }^{5}$ neuroleptic, ${ }^{6}$ and cardiac stimulant ${ }^{7}$ activities. In our earlier studies, we reported that 6-alkyloxyl-3,4-dihydro-2(1H)-quinolinones show good anticonvulsant activities in the MES and PZT-induced seizure test. ${ }^{8}$

In the followed studies, we synthesized several $[1,2,4]$ triazolo[4,3-a]quinoline derivatives and tested their anticonvulsant activities. We found that 1-substituted-7-benzyloxy-4,5dihydro-[1,2,4] triazolo[4,3-a]quinolines, ${ }^{9}$ 7-alkoxyl-4,5-dihydro-[1,2,4]triazolo[4,3-a]quinolines ${ }^{10}$ and 5-alkoxy-[1,2,4] triazolo[4,3-a]quinolines [In press] had significant anticonvulsant activities.

As part of our continuous effort to find better anticonvulsant agents in this area, a series of new 5-alkoxy-tetrazolo[1,5-a] quinolines were synthesized as outlined in Scheme 1. Anticonvulsant activities and neurotoxicity of the synthesized com- pounds were determined by the maximal electroshock (MES) test and rotarod test according to the protocols for phase I tests of the antiepileptic drug development (ADD) program, which were developed by the National Institute of Neurological Disorders and Stroke. ${ }^{11-12}$

We also evaluated the antidepressant activities of the synthesized compounds using Porsolt's behavioral despair test/ forced swimming test (FST). ${ }^{13}$ As the monoaminergic system is one of the most important targets in the therapies for depression, ${ }^{14,15}$ we used two behavioral models to investigate the possible mechanism involving monoaminergic participation in the antidepressant-like effect of the single compound, $\mathbf{4 k}$, in this study.

\section{Experimental}

Chemistry. Melting points were determined in open capillary tubes and were uncorrected. IR spectra were recorded (in $\mathrm{KBr}$ ) on a FT-IR1730. ${ }^{1} \mathrm{H}-\mathrm{NMR}$ spectra were measured on an AV-300 (Bruker, Switzerland, and all chemical shifts were given in ppm<smiles>O=c1cc(O)c2ccccc2[nH]1</smiles><smiles>[R]C(C)OCC</smiles><smiles>[R7]c1cc(=O)[nH]c2ccccc12</smiles>

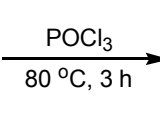<smiles>[R7]Oc1cc(Cl)nc2ccccc12</smiles><smiles>CC(C)(C)O[Na]</smiles>

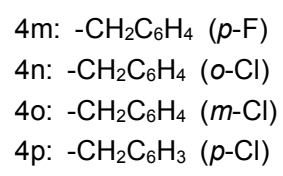

$$
\begin{aligned}
& \text { 4e: } n-\mathrm{C}_{5} \mathrm{H}_{11} \\
& \text { 4f: } n-\mathrm{C}_{6} \mathrm{H}_{13} \\
& \text { 4g: } n-\mathrm{C}_{7} \mathrm{H}_{15} \\
& \text { 4h: } n-\mathrm{C}_{8} \mathrm{H}_{17}
\end{aligned}
$$

$$
\begin{aligned}
& \text { 4q: }-\mathrm{CH}_{2} \mathrm{C}_{6} \mathrm{H}_{4}(o-\mathrm{Br}) \\
& \text { 4r: }-\mathrm{CH}_{2} \mathrm{C}_{6} \mathrm{H}_{4}(p-\mathrm{Br}) \\
& \text { 4s: }-\mathrm{CH}_{2} \mathrm{C}_{6} \mathrm{H}_{4}\left(p-\mathrm{CH}_{3}\right)
\end{aligned}
$$


relative to tetramethysilane. Mass spectra were measured on an HP1100LC (Agilent Technologies, USA). Elemental analyses were performed on a 204Q CHN (Perkin Elmer, USA). Microanalyses of $\mathrm{C}, \mathrm{N}$, and $\mathrm{H}$ were performed using a Heraeus CHN Rapid Analyzer. The major chemicals were purchased from Sigma-Aldrich Corporation.

Based on previous studies in our laboratory, we designed and prepared a series of 5-alkoxy-tetrazolo[1,5-a]quinolines (4a-4s). Target compounds $\mathbf{4 a - 4 s}$ were synthesized according to Scheme 1. The starting material 4-hydroxyquinolin-2(1H)one reacted with the appropriate alkyl halide or benzyl chloride to obtain 4-alkoxyquinolinones (2a-2s) in DMF with stirring and refluxing. ${ }^{16}$ Compounds 2 a-2s reacted further with $\mathrm{POCl}_{3}$ at $80{ }^{\circ} \mathrm{C}$ to yield 2-chloro-4-alkoxyquinolines (3a-3s). Compounds 3a-3s reacted with sodium azide in DMSO to obtain the target compounds, $\mathbf{4 a - 4 s}$. $^{17}$

General procedures for the synthesis of the compounds 2 a2s: A mixture of 4-hydroxyquinolin-2(1H)-one (1.00 g, 6.2 $\mathrm{mmol})$ and $\mathrm{K}_{2} \mathrm{CO}_{3}(1.71 \mathrm{~g}, 12.4 \mathrm{mmol})$ in DMF $(50 \mathrm{~mL})$ was heated at $90{ }^{\circ} \mathrm{C}$ for $2 \mathrm{~h}$. The mixture was cooled to room temperature, then appropriate alkyl halides/benzyl chlorides $(6.2$ mmol) was added, and the mixture was heated at $50 \sim 90{ }^{\circ} \mathrm{C}$ for $4 \sim 12 \mathrm{~h}$ (TLC monitoring). The mixture was poured into ice water $(200 \mathrm{~mL})$; the precipitate that separated was collected by filtration, washed with water and ethyl acetate and then dried to give $\mathbf{2 a - 2 s}$ with enough purity.

General procedures for the synthesis of the compounds 3a3s: A solution of 2a-2s (5 mmol) in appropriate $\mathrm{POCl}_{3}$ and triethylamine $(5 \mathrm{mmol})$ was heated for $3 \mathrm{~h}$ at $80{ }^{\circ} \mathrm{C}$. After removing the solvent under reduced pressure, the residue was dissolved in dichloromethane $(40 \mathrm{~mL})$, washed two times with saturated aqueous $\mathrm{NaHCO}_{3}$ and once with saturated aqueous $\mathrm{NaCl}$. The dichloromethane layer was dried over anhydrous $\mathrm{MgSO}_{4}$. Evaporation of the solvents gave $\mathbf{2 a - 2} \mathbf{u}$ that was pure enough.

General procedures for the synthesis of the compounds $4 a-$ 4s: To a solution of 3a-3s (5 mmol) in DMSO (50 mL), sodium azide $(0.49 \mathrm{~g}, 7.5 \mathrm{mmol})$ was added portionwise. The reaction mixture was stirred at $40 \sim 80{ }^{\circ} \mathrm{C}$ for $24 \mathrm{~h}$. The white precipitate formed was filtered, washed with water and crystallized from ethyl acetate and dichloromethane. The yield, melting point and spectral data of each compound were given below.

5-Methoxytetrazolo[1,5-a]quinoline (4a): $\mathrm{mp} 222 \sim 224{ }^{\circ} \mathrm{C}$; yield $81.2 \% .{ }^{1} \mathrm{H}-\mathrm{NMR}\left(\mathrm{CDCl}_{3}, 300 \mathrm{MHz}\right): \delta 4.14\left(\mathrm{~s}, 3 \mathrm{H}, \mathrm{OCH}_{3}\right)$, $7.01(\mathrm{~s}, 1 \mathrm{H}, \mathrm{CH}=), 7.68(\mathrm{t}, 1 \mathrm{H}, J=7.7 \mathrm{~Hz}, \mathrm{H}-7), 7.86(\mathrm{t}, 1 \mathrm{H}, J=$ $7.7 \mathrm{~Hz}, \mathrm{H}-8), 8.26$ (d, $1 \mathrm{H}, J=8.3 \mathrm{~Hz}, \mathrm{H}-9), 8.60$ (d, $1 \mathrm{H}, J=8.2$ $\mathrm{Hz}, \mathrm{H}-6)$. IR (KBr) cm ${ }^{-1}$ : 1127, 1224 (C-O-C), $1617(\mathrm{C}=\mathrm{N})$. MS $(\mathrm{m} / \mathrm{z})$ : $201(\mathrm{M}+1)$. Anal. Calcd. for C10H8N4O: C, 59.99; H, 4.03; N, 27.99. Found: C, 60.11; H, 4.15; N, 27.85.

5-Ethoxytetrazolo[1,5-a]quinoline (4b): $\mathrm{mp} 216 \sim 218^{\circ} \mathrm{C}$; yield $74.5 \%$. ${ }^{1} \mathrm{H}-\mathrm{NMR}\left(\mathrm{CDCl}_{3}, 300 \mathrm{MHz}\right): \delta 1.65$ (t, $3 \mathrm{H}, J=$ $\left.6.98 \mathrm{~Hz}, \mathrm{CH}_{3}\right), 4.36$ (q, 2H, J=6.97 Hz, $\left.\mathrm{OCH}_{2}\right), 7.02(\mathrm{~s}, 1 \mathrm{H}$, $\mathrm{CH}=), 7.69(\mathrm{t}, 1 \mathrm{H}, J=7.7 \mathrm{~Hz}, \mathrm{H}-7), 7.87$ (t, $1 \mathrm{H}, J=7.5 \mathrm{~Hz}, \mathrm{H}-8)$, 8.32 (d, 1H, $J=8.1 \mathrm{~Hz}, \mathrm{H}-9), 8.63$ (d, 1H, $J=8.2 \mathrm{~Hz}, \mathrm{H}-6)$. IR $(\mathrm{KBr}) \mathrm{cm}^{-1}: 1124,1226(\mathrm{C}-\mathrm{O}-\mathrm{C}), 1617(\mathrm{C}=\mathrm{N})$. MS $(\mathrm{m} / \mathrm{z}): 215$ $(\mathrm{M}+1)$. Anal. Calcd. for C11H10N4O: C, 61.67; H, 4.71; N, 26.15. Found: C, 61.85; H, 4.74; N, 26.02.

5-Propoxytetrazolo[1,5-a]quinoline (4c): $\mathrm{mp} 204 \sim 207^{\circ} \mathrm{C}$; yield 56.5\%. ${ }^{1} \mathrm{H}-\mathrm{NMR}\left(\mathrm{CDCl}_{3}, 300 \mathrm{MHz}\right): \delta 1.16(\mathrm{t}, 3 \mathrm{H}, J=$ $\left.7.41 \mathrm{~Hz}, \mathrm{CH}_{3}\right), 2.01\left(\mathrm{~m}, 2 \mathrm{H}, J=6.98 \mathrm{~Hz}, \mathrm{CH}_{2}\right), 4.21(\mathrm{t}, 2 \mathrm{H}, J=$ $\left.6.38 \mathrm{~Hz}, \mathrm{OCH}_{2}\right), 6.96(\mathrm{~s}, 1 \mathrm{H}, \mathrm{CH}=), 7.65(\mathrm{t}, 1 \mathrm{H}, J=7.7 \mathrm{~Hz}, \mathrm{H}-7)$, 7.83 (t, $1 \mathrm{H}, J=7.8 \mathrm{~Hz}, \mathrm{H}-8), 8.26(\mathrm{~d}, 1 \mathrm{H}, J=8.2 \mathrm{~Hz}, \mathrm{H}-9), 8.57$ (d, $1 \mathrm{H}, J=8.3 \mathrm{~Hz}, \mathrm{H}-6)$. IR (KBr) cm ${ }^{-1}: 1125,1228$ (C-O-C), $1618(\mathrm{C}=\mathrm{N})$. MS $(\mathrm{m} / \mathrm{z}): 229(\mathrm{M}+1)$. Anal. Calcd. for $\mathrm{C} 12 \mathrm{H} 12$ N4O: C, 63.15; H, 5.30; N, 24.55. Found: C, 63.25; H,5.43; N, 24.38 .

5-Butoxytetrazolo[1,5-a]quinoline (4d): $\mathrm{mp} 178 \sim 181{ }^{\circ} \mathrm{C}$; yield $64.6 \%$. ${ }^{1} \mathrm{H}-\mathrm{NMR}\left(\mathrm{CDCl}_{3}, 300 \mathrm{MHz}\right): \delta 1.05$ (t, $3 \mathrm{H}, J=$ $\left.7.38 \mathrm{~Hz}, \mathrm{CH}_{3}\right), 1.59\left(\mathrm{~m}, 2 \mathrm{H}, J=7.46 \mathrm{~Hz}, \mathrm{CH}_{2}\right), 1.97(\mathrm{~m}, 2 \mathrm{H}$, $\left.J=6.94 \mathrm{~Hz}, \mathrm{CH}_{2}\right), 4.26\left(\mathrm{t}, 2 \mathrm{H}, J=6.33 \mathrm{~Hz}, \mathrm{OCH}_{2}\right), 6.98(\mathrm{~s}, 1 \mathrm{H}$, $\mathrm{CH}=), 7.67(\mathrm{t}, 1 \mathrm{H}, J=7.7 \mathrm{~Hz}, \mathrm{H}-7), 7.85$ (t, $1 \mathrm{H}, J=7.8 \mathrm{~Hz}, \mathrm{H}-8)$, 8.27 (d, $1 \mathrm{H}, J=8.1 \mathrm{~Hz}, \mathrm{H}-9), 8.59$ (d, $1 \mathrm{H}, J=8.3 \mathrm{~Hz}, \mathrm{H}-6)$. IR $(\mathrm{KBr}) \mathrm{cm}^{-1}: 1122,1229(\mathrm{C}-\mathrm{O}-\mathrm{C}), 1616(\mathrm{C}=\mathrm{N}) . \mathrm{MS}(\mathrm{m} / \mathrm{z}): 243$ $(\mathrm{M}+1)$. Anal. Calcd. for C13H14N4O: C, 64.45; H, 5.82; N, 23.13. Found: C, 64.65; H,5.88; N, 23.31.

5-(Pentyloxy)tetrazolo[1,5-a]quinoline (4e): mp $158 \sim 160$ ${ }^{\circ} \mathrm{C}$; yield 78.2\%. ${ }^{1} \mathrm{H}-\mathrm{NMR}\left(\mathrm{CDCl}_{3}, 300 \mathrm{MHz}\right): \delta 0.99(\mathrm{t}, 3 \mathrm{H}$, $\left.J=7.13 \mathrm{~Hz}, \mathrm{CH}_{3}\right), 1.43-1.61\left(\mathrm{~m}, 4 \mathrm{H}, \mathrm{CH}_{2}\right), 2.01$ (m, $2 \mathrm{H}, J=6.92$ $\left.\mathrm{Hz}, \mathrm{CH}_{2}\right), 4.28\left(\mathrm{t}, 2 \mathrm{H}, J=6.38 \mathrm{~Hz}, \mathrm{OCH}_{2}\right), 7.00(\mathrm{~s}, 1 \mathrm{H}, \mathrm{CH}=)$, 7.70 (t, $1 \mathrm{H}, J=7.7 \mathrm{~Hz}, \mathrm{H}-7), 7.88(\mathrm{t}, 1 \mathrm{H}, J=7.6 \mathrm{~Hz}, \mathrm{H}-8), 8.30$ (d, $1 \mathrm{H}, J=8.2 \mathrm{~Hz}, \mathrm{H}-9), 8.63$ (d, 1H, $J=8.3 \mathrm{~Hz}, \mathrm{H}-6)$. IR (KBr) $\mathrm{cm}^{-1}: 1124,1226(\mathrm{C}-\mathrm{O}-\mathrm{C}), 1616(\mathrm{C}=\mathrm{N})$. MS $(\mathrm{m} / \mathrm{z}): 257(\mathrm{M}+1)$. Anal. Calcd. for C14H16N4O: C, 65.61; H, 6.29; N, 21.86. Found: C, 65.65; H,6.36; N, 21.59.

5-(Hexyloxy)tetrazolo[1,5-a]quinoline (4f): mp $148 \sim 150$ ${ }^{0} \mathrm{C}$; yield 67.8\% ${ }^{1} \mathrm{H}-\mathrm{NMR}\left(\mathrm{CDCl}_{3}, 300 \mathrm{MHz}\right): \delta 0.92(\mathrm{t}, 3 \mathrm{H}$, $\left.J=6.87 \mathrm{~Hz}, \mathrm{CH}_{3}\right), 1.37-1.59\left(\mathrm{~m}, 6 \mathrm{H}, \mathrm{CH}_{2}\right), 1.98(\mathrm{~m}, 2 \mathrm{H}, J=6.97$ $\left.\mathrm{Hz}, \mathrm{CH}_{2}\right), 4.25\left(\mathrm{t}, 2 \mathrm{H}, J=6.38 \mathrm{~Hz}, \mathrm{OCH}_{2}\right), 6.97$ (s, $\left.1 \mathrm{H}, \mathrm{CH}=\right)$, $7.66(\mathrm{t}, 1 \mathrm{H}, J=7.6 \mathrm{~Hz}, \mathrm{H}-7), 7.84$ (t, $1 \mathrm{H}, J=7.4 \mathrm{~Hz}, \mathrm{H}-8), 8.26$ (d, $1 \mathrm{H}, J=8.1 \mathrm{~Hz}, \mathrm{H}-9), 8.58$ (d, 1H, $J=8.3 \mathrm{~Hz}, \mathrm{H}-6)$. IR (KBr) $\mathrm{cm}^{-1}: 1129,1225(\mathrm{C}-\mathrm{O}-\mathrm{C}), 1617(\mathrm{C}=\mathrm{N})$. MS $(\mathrm{m} / \mathrm{z}): 271(\mathrm{M}+1)$. Anal. Calcd. for C15H18N4O: C, 66.64; H, 6.71; N, 20.73. Found: C, 66.78; H,6.85; N, 20.51.

5-(Heptyloxy)tetrazolo[1,5-a]quinoline (4g): mp $145 \sim 147$ ${ }^{\circ} \mathrm{C}$; yield 78.0\%. ${ }^{1} \mathrm{H}-\mathrm{NMR}\left(\mathrm{CDCl}_{3}, 300 \mathrm{MHz}\right): \delta 0.90(\mathrm{t}, 3 \mathrm{H}$, $\left.J=6.78 \mathrm{~Hz}, \mathrm{CH}_{3}\right), 1.35-1.57\left(\mathrm{~m}, 8 \mathrm{H}, \mathrm{CH}_{2}\right), 1.98(\mathrm{~m}, 2 \mathrm{H}, J=$ $\left.7.06 \mathrm{~Hz}, \mathrm{CH}_{2}\right), 4.25$ (t, $\left.2 \mathrm{H}, J=6.24 \mathrm{~Hz}, \mathrm{OCH}_{2}\right), 6.97(\mathrm{~s}, 1 \mathrm{H}$, $\mathrm{CH}=), 7.67(\mathrm{t}, 1 \mathrm{H}, J=7.6 \mathrm{~Hz}, \mathrm{H}-7), 7.85$ (t, $1 \mathrm{H}, J=7.5 \mathrm{~Hz}, \mathrm{H}-8)$, 8.27 (d, $1 \mathrm{H}, J=8.0 \mathrm{~Hz}, \mathrm{H}-9), 8.59$ (d, $1 \mathrm{H}, J=8.2 \mathrm{~Hz}, \mathrm{H}-6)$. IR $(\mathrm{KBr}) \mathrm{cm}^{-1}: 1130,1221(\mathrm{C}-\mathrm{O}-\mathrm{C}), 1618(\mathrm{C}=\mathrm{N}) . \mathrm{MS}(\mathrm{m} / \mathrm{z}): 285$ $(\mathrm{M}+1)$. Anal. Calcd. for C16H20N4O: C, 67.58; H, 7.09; N, 19.70. Found: C, 67.67; H,7.23; N, 19.59.

5-(Octyloxy)tetrazolo[1,5-a]quinoline (4h): mp 149 152 ${ }^{\circ} \mathrm{C}$; yield $83.7 \%$. ${ }^{1} \mathrm{H}-\mathrm{NMR}\left(\mathrm{CDCl}_{3}, 300 \mathrm{MHz}\right): \delta 0.89(\mathrm{t}, 3 \mathrm{H}$, $\left.J=6.61 \mathrm{~Hz}, \mathrm{CH}_{3}\right), 1.32-1.58\left(\mathrm{~m}, 10 \mathrm{H}, \mathrm{CH}_{2}\right), 1.99(\mathrm{~m}, 2 \mathrm{H}, J=$ $\left.7.32 \mathrm{~Hz}, \mathrm{CH}_{2}\right), 4.27$ (t, $\left.2 \mathrm{H}, J=6.41 \mathrm{~Hz}, \mathrm{OCH}_{2}\right), 6.70(\mathrm{~s}, 1 \mathrm{H}$, $\mathrm{CH}=), 7.69$ (t, 1H, $J=7.7 \mathrm{~Hz}, \mathrm{H}-7), 7.87$ (t, $1 \mathrm{H}, J=7.7 \mathrm{~Hz}, \mathrm{H}-8)$, $8.30(\mathrm{~d}, 1 \mathrm{H}, J=8.2 \mathrm{~Hz}, \mathrm{H}-9), 8.63$ (d, $1 \mathrm{H}, J=8.4 \mathrm{~Hz}, \mathrm{H}-6)$. IR $(\mathrm{KBr}) \mathrm{cm}^{-1}: 1128,1225(\mathrm{C}-\mathrm{O}-\mathrm{C}), 1616(\mathrm{C}=\mathrm{N}) . \mathrm{MS}(\mathrm{m} / \mathrm{z}): 299$ $(\mathrm{M}+1)$. Anal. Calcd. for C17H22N4O: C, 68.43; H, 7.43; N, 18.78. Found: C, 68.67; H,7.61; N, 18.63.

5-(Dodecyloxy)tetrazolo[1,5-a]quinoline (4i): mp 108 110 ${ }^{\circ} \mathrm{C}$; yield 88.6\% ${ }^{1} \mathrm{H}-\mathrm{NMR}\left(\mathrm{CDCl}_{3}, 300 \mathrm{MHz}\right): \delta 0.88(\mathrm{t}, 3 \mathrm{H}$, $\left.J=6.77 \mathrm{~Hz}, \mathrm{CH}_{3}\right), 1.27-1.57\left(\mathrm{~m}, 18 \mathrm{H}, \mathrm{CH}_{2}\right), 1.99$ (m, 2H, $J=$ $\left.6.95 \mathrm{~Hz}, \mathrm{CH}_{2}\right), 4.27$ (t, $\left.2 \mathrm{H}, J=6.36 \mathrm{~Hz}, \mathrm{OCH}_{2}\right), 7.00(\mathrm{~s}, 1 \mathrm{H}$, $\mathrm{CH}=), 7.69$ (t, 1H, J=7.8 Hz, H-7), 7.87 (t, 1H, $J=7.3 \mathrm{~Hz}, \mathrm{H}-8)$, 
$8.30(\mathrm{~d}, 1 \mathrm{H}, J=8.2 \mathrm{~Hz}, \mathrm{H}-9), 8.63(\mathrm{~d}, 1 \mathrm{H}, J=8.2 \mathrm{~Hz}, \mathrm{H}-6)$. IR $(\mathrm{KBr}) \mathrm{cm}^{-1}: 1131,1222(\mathrm{C}-\mathrm{O}-\mathrm{C}), 1615(\mathrm{C}=\mathrm{N})$. MS $(\mathrm{m} / \mathrm{z}): 355$ $(\mathrm{M}+1)$. Anal. Calcd. for C21H30N4O: C, 71.15; H, 8.53; N, 15.80. Found: C, 71.32; H, 8.64; N, 15.59.

5-(Benzyloxy)tetrazolo[1,5-a]quinoline (4j): $\mathrm{mp} 232 \sim 233$ ${ }^{\circ} \mathrm{C}$; yield 73.8\%. ${ }^{1} \mathrm{H}-\mathrm{NMR}\left(\mathrm{CDCl}_{3}, 300 \mathrm{MHz}\right): \delta 5.37(\mathrm{~s}, 2 \mathrm{H}$, $\left.\mathrm{OCH}_{2}\right), 7.11(\mathrm{~s}, 1 \mathrm{H}, \mathrm{CH}=), 7.41-7.54(\mathrm{~m}, 5 \mathrm{H}, \mathrm{Ar}-\mathrm{H}), 7.69(\mathrm{t}, 1 \mathrm{H}$, $J=7.8 \mathrm{~Hz}, \mathrm{H}-7), 7.86(\mathrm{t}, 1 \mathrm{H}, J=7.7 \mathrm{~Hz}, \mathrm{H}-8), 8.35$ (d, $1 \mathrm{H}, J=$ $8.3 \mathrm{~Hz}, \mathrm{H}-9), 8.64$ (d, $1 \mathrm{H}, J=8.3 \mathrm{~Hz}, \mathrm{H}-6)$. IR (KBr) cm ${ }^{-1}$ : 1128 , $1226(\mathrm{C}-\mathrm{O}-\mathrm{C}), 1621(\mathrm{C}=\mathrm{N})$. MS $(\mathrm{m} / \mathrm{z})$ : $277(\mathrm{M}+1)$. Anal. Calcd. for C16H12N4O: C, 69.55; H, 4.38; N, 20.28. Found: C, 69.63; $\mathrm{H}, 4.45 ; \mathrm{N}, 20.21$.

5-(2-Fluorobenzyloxy)tetrazolo[1,5-a]quinoline (4k): $\mathrm{mp}$ $243 \sim 245{ }^{\circ} \mathrm{C}$; yield 64.3\%. ${ }^{1} \mathrm{H}-\mathrm{NMR}\left(\mathrm{CDCl}_{3}, 300 \mathrm{MHz}\right): \delta 5.44$ $\left(\mathrm{s}, 2 \mathrm{H}, \mathrm{OCH}_{2}\right), 7.15(\mathrm{~s}, 1 \mathrm{H}, \mathrm{CH}=), 7.21(\mathrm{t}, 1 \mathrm{H}, J=7.9 \mathrm{~Hz}, \mathrm{Ar}-\mathrm{H})$, $7.25(\mathrm{~d}, 1 \mathrm{H}, J=8.2 \mathrm{~Hz}, \mathrm{Ar}-\mathrm{H}), 7.42\left(\mathrm{dd}, 1 \mathrm{H}, J_{1}=6.1 \mathrm{~Hz}, J_{2}=\right.$ $13.3 \mathrm{~Hz}, \mathrm{Ar}-\mathrm{H}), 7.56$ (t, 1H, $J=7.4 \mathrm{~Hz}, \mathrm{Ar}-\mathrm{H}), 7.69$ (t, $1 \mathrm{H}, J=$ $7.7 \mathrm{~Hz}, \mathrm{H}-7), 7.88$ (t, $1 \mathrm{H}, J=7.8 \mathrm{~Hz}, \mathrm{H}-8), 8.31$ (d, $1 \mathrm{H}, J=8.3$ $\mathrm{Hz}, \mathrm{H}-9), 8.64$ (d, 1H, J=8.2 Hz, H-6). IR (KBr) cm ${ }^{-1}$ : 1128 , $1227(\mathrm{C}-\mathrm{O}-\mathrm{C}), 1622(\mathrm{C}=\mathrm{N})$. MS ( $\mathrm{m} / \mathrm{z}): 295(\mathrm{M}+1)$. Anal. Calcd. for C16H11FN4O: C, 65.30; H, 3.77; N, 19.04. Found: C, 65.43; $\mathrm{H}, 3.86 ; \mathrm{N}, 18.92$.

5-(3-Fluorobenzyloxy)tetrazolo[1,5-a]quinoline (4I): $\mathrm{mp}$ $267 \sim 269{ }^{\circ} \mathrm{C}$; yield $78.4 \%$. ${ }^{1} \mathrm{H}-\mathrm{NMR}\left(\mathrm{CDCl}_{3}, 300 \mathrm{MHz}\right): \delta 5.36$ $\left(\mathrm{s}, 2 \mathrm{H}, \mathrm{OCH}_{2}\right), 7.16(\mathrm{~s}, 1 \mathrm{H}, \mathrm{CH}=), 7.05(\mathrm{t}, 1 \mathrm{H}, J=8.6 \mathrm{~Hz}$, Ar-H), $7.21(\mathrm{~d}, 1 \mathrm{H}, J=9.7 \mathrm{~Hz}, \mathrm{Ar}-\mathrm{H}), 7.27(\mathrm{~d}, 1 \mathrm{H}, J=7.1 \mathrm{~Hz}$, Ar-H), $7.36\left(\mathrm{dd}, 1 \mathrm{H}, J_{1}=7.8 \mathrm{~Hz}, J_{2}=13.7 \mathrm{~Hz}, \mathrm{Ar}-\mathrm{H}\right), 7.67(\mathrm{t}$, $1 \mathrm{H}, J=7.7 \mathrm{~Hz}, \mathrm{H}-7), 7.85$ (t, $1 \mathrm{H}, J=7.4 \mathrm{~Hz}, \mathrm{H}-8), 8.27$ (d, 1H, $J=8.2 \mathrm{~Hz}, \mathrm{H}-9), 8.52$ (d, $1 \mathrm{H}, J=8.3 \mathrm{~Hz}, \mathrm{H}-6) . \mathrm{IR}(\mathrm{KBr}) \mathrm{cm}^{-1}$ : 1128, 1228 (C-O-C), $1622(\mathrm{C}=\mathrm{N})$. MS ( $\mathrm{m} / \mathrm{z})$ : $295(\mathrm{M}+1)$. Anal. Calcd. for C16H11FN4O: C, 65.30; H, 3.77; N, 19.04. Found: C, 65.45; H, 3.89; N, 18.91 .

5-(4-Fluorobenzyloxy)tetrazolo[1,5-a]quinoline (4m): $\mathrm{mp}$ $262 \sim 264{ }^{\circ} \mathrm{C}$; yield 69.8\%. ${ }^{1} \mathrm{H}-\mathrm{NMR}\left(\mathrm{CDCl}_{3}, 300 \mathrm{MHz}\right): \delta$ $5.33\left(\mathrm{~s}, 2 \mathrm{H}, \mathrm{OCH}_{2}\right), 7.15(\mathrm{~s}, 1 \mathrm{H}, \mathrm{CH}=), 7.14\left(\mathrm{dd}, 2 \mathrm{H}, J_{1}=8.4\right.$ $\left.\mathrm{Hz}, J_{2}=15.2 \mathrm{~Hz}, \mathrm{Ar}-\mathrm{H}\right), 7.51\left(\mathrm{dd}, 1 \mathrm{H}, J_{1}=8.4 \mathrm{~Hz}, J_{2}=5.4 \mathrm{~Hz}\right.$, Ar-H), 7.69 (t, 1H, $J=7.7 \mathrm{~Hz}, \mathrm{H}-7), 7.89$ (t, $1 \mathrm{H}, J=7.6 \mathrm{~Hz}$, $\mathrm{H}-8), 8.31$ (d, $1 \mathrm{H}, J=8.3 \mathrm{~Hz}, \mathrm{H}-9), 8.65$ (d, $1 \mathrm{H}, J=8.3 \mathrm{~Hz}$, $\mathrm{H}-6)$. IR (KBr) cm ${ }^{-1}: 1128,1227(\mathrm{C}-\mathrm{O}-\mathrm{C}), 1623(\mathrm{C}=\mathrm{N})$. MS $(\mathrm{m} / \mathrm{z})$ : $295(\mathrm{M}+1)$. Anal. Calcd. for C16H11FN4O: C, 65.30; H, 3.77; N, 19.04. Found: C, 65.47; H, 3.84; N, 18.96 .

5-(2-Chlorobenzyloxy)tetrazolo[1,5-a]quinoline (4n): $\mathrm{mp}$ $204 \sim 206{ }^{\circ} \mathrm{C}$; yield 71.7\%. ${ }^{1} \mathrm{H}-\mathrm{NMR}\left(\mathrm{CDCl}_{3}, 300 \mathrm{MHz}\right): \delta 5.47$ $\left(\mathrm{s}, 2 \mathrm{H}, \mathrm{OCH}_{2}\right), 7.13(\mathrm{~s}, 1 \mathrm{H}, \mathrm{CH}=), 7.26-7.37$ (m, 2H, Ar- H), 7.46-7.61 (m, 2H, Ar-H), $7.71(\mathrm{t}, 1 \mathrm{H}, J=7.6 \mathrm{~Hz}, \mathrm{H}-7), 7.89$ (t, $1 \mathrm{H}, J=7.6 \mathrm{~Hz}, \mathrm{H}-8), 8.35$ (d, $1 \mathrm{H}, J=8.3 \mathrm{~Hz}, \mathrm{H}-9), 8.65$ (d, $1 \mathrm{H}, J=8.2 \mathrm{~Hz}, \mathrm{H}-6)$. IR (KBr) cm $\mathrm{cm}^{-1}: 1131,1230$ (C-O-C), 1624 $(\mathrm{C}=\mathrm{N})$. $\mathrm{MS}(\mathrm{m} / \mathrm{z}): 312(\mathrm{M}+1)$. Anal. Calcd. for C16H11CIN4O: C, 61.84; H, 3.57; N, 18.03. Found: C, 61.97; H, 3.69; N, 17.91.

5-(3-Chlorobenzyloxy)tetrazolo[1,5-a]quinoline (4o): $\mathrm{mp}$ $265 \sim 267{ }^{\circ} \mathrm{C}$; yield $79.4 \%$. ${ }^{1} \mathrm{H}-\mathrm{NMR}\left(\mathrm{CDCl}_{3}, 300 \mathrm{MHz}\right): \delta 5.35$ (s, 2H, $\left.\mathrm{OCH}_{2}\right), 7.09$ (s, 1H, CH=), 7.39-7.41 (m, 3H, Ar-H), $7.53(\mathrm{~s}, 1 \mathrm{H}, \mathrm{Ar}-\mathrm{H}), 7.72(\mathrm{t}, 1 \mathrm{H}, J=8.0 \mathrm{~Hz}, \mathrm{H}-7), 7.90(\mathrm{t}, 1 \mathrm{H}$, $J=7.8 \mathrm{~Hz}, \mathrm{H}-8), 8.34$ (d, 1H, $J=8.0 \mathrm{~Hz}, \mathrm{H}-9), 8.65$ (d, $1 \mathrm{H}, J=$ $8.4 \mathrm{~Hz}, \mathrm{H}-6), 7.72-8.67$ (m, 4H, Ar-H). IR (KBr) cm ${ }^{-1}$ : 1130 , $1228(\mathrm{C}-\mathrm{O}-\mathrm{C}), 1625(\mathrm{C}=\mathrm{N})$. MS $(\mathrm{m} / \mathrm{z}): 312(\mathrm{M}+1)$. Anal. Calcd. for C16H11CIN4O: C, 61.84; H, 3.57; N, 18.03. Found: C, $61.94 ; \mathrm{H}, 3.66 ; \mathrm{N}, 17.89$.
5-(4-Chlorobenzyloxy)tetrazolo[1,5-a]quinoline (4p): $\mathrm{mp}$ $285 \sim 287^{\circ} \mathrm{C}$; yield 69.8\%. ${ }^{1} \mathrm{H}-\mathrm{NMR}\left(\mathrm{CDCl}_{3}, 300 \mathrm{MHz}\right): \delta 5.34$ $\left(\mathrm{s}, 2 \mathrm{H}, \mathrm{OCH}_{2}\right), 7.09(\mathrm{~s}, 1 \mathrm{H}, \mathrm{CH}=), 7.44(\mathrm{~d}, 2 \mathrm{H}, J=8.9 \mathrm{~Hz}$, Ar-H), 7.48 (d, 2H, $J=8.9 \mathrm{~Hz}, \operatorname{Ar}-\mathrm{H}), 7.70(\mathrm{t}, 1 \mathrm{H}, J=7.7 \mathrm{~Hz}$, H-7), 7.90 (t, $1 \mathrm{H}, J=7.5 \mathrm{~Hz}, \mathrm{H}-8), 8.31$ (d, $1 \mathrm{H}, J=8.2 \mathrm{~Hz}$, $\mathrm{H}-9), 8.65$ (d, $1 \mathrm{H}, J=8.3 \mathrm{~Hz}, \mathrm{H}-6)$. IR (KBr) cm ${ }^{-1}: 1131,1230$ (C-O-C), $1624(\mathrm{C}=\mathrm{N})$. MS $(\mathrm{m} / \mathrm{z})$ : $312(\mathrm{M}+1)$. Anal. Calcd. for C16H11CIN4O: C, 61.84; H, 3.57; N, 18.03. Found: C, 61.99; H, 3.72; N, 17.91.

5-(2-Bromobenzyloxy)tetrazolo[1,5-a]quinoline (4q): $\mathrm{mp}$ $220 \sim 222{ }^{\circ} \mathrm{C}$; yield 74.2\%. ${ }^{1} \mathrm{H}-\mathrm{NMR}\left(\mathrm{CDCl}_{3}, 300 \mathrm{MHz}\right): \delta 5.45$ $\left(\mathrm{s}, 2 \mathrm{H}, \mathrm{OCH}_{2}\right), 7.12(\mathrm{~s}, 1 \mathrm{H}, \mathrm{CH}=), 7.29(\mathrm{t}, 1 \mathrm{H}, J=8.5 \mathrm{~Hz}, \mathrm{Ar}-\mathrm{H})$, 7.40 (t, 1H, $J=7.2 \mathrm{~Hz}, \mathrm{Ar}-\mathrm{H}), 7.58$ (d, 1H, $J=7.5 \mathrm{~Hz}, \mathrm{Ar}-\mathrm{H})$, $7.68(\mathrm{~d}, 1 \mathrm{H}, J=7.5 \mathrm{~Hz}, \mathrm{Ar}-\mathrm{H}), 7.71(\mathrm{t}, 1 \mathrm{H}, J=7.0 \mathrm{~Hz}, \mathrm{H}-7)$, 7.90 (t, 1H, $J=7.8 \mathrm{~Hz}, \mathrm{H}-8), 8.37$ (d, 1H, $J=8.1 \mathrm{~Hz}, \mathrm{H}-9), 8.65$ (d, $1 \mathrm{H}, J=8.3 \mathrm{~Hz}, \mathrm{H}-6)$. IR (KBr) cm ${ }^{-1}: 1130,1229$ (C-O-C), $1622(\mathrm{C}=\mathrm{N})$. MS $(\mathrm{m} / \mathrm{z}): 356(\mathrm{M}+1)$. Anal. Calcd. for $\mathrm{C} 16 \mathrm{H} 11$ BrN4O: C, 54.10; H, 3.12; N, 15.77. Found: C, 54.27; H, 3.32; $\mathrm{N}, 15.53$.

5-(4-Bromobenzyloxy)tetrazolo[1,5-a]quinoline (4r): $\mathrm{mp}$ $286 \sim 287^{\circ} \mathrm{C}$; yield $80.2 \% .{ }^{1} \mathrm{H}-\mathrm{NMR}\left(\mathrm{CDCl}_{3}, 300 \mathrm{MHz}\right): \delta 5.32$ $\left(\mathrm{s}, 2 \mathrm{H}, \mathrm{OCH}_{2}\right), 7.09(\mathrm{~s}, 1 \mathrm{H}, \mathrm{CH}=), 7.41(\mathrm{~d}, 2 \mathrm{H}, J=8.2 \mathrm{~Hz}, \mathrm{Ar}-\mathrm{H})$, 7.60 (d, 2H, $J=8.2 \mathrm{~Hz}, \mathrm{Ar}-\mathrm{H}), 7.70$ (t, $1 \mathrm{H}, J=7.8 \mathrm{~Hz}, \mathrm{H}-7)$, 7.90 (t, $1 \mathrm{H}, J=7.8 \mathrm{~Hz}, \mathrm{H}-8), 8.31$ (d, $1 \mathrm{H}, J=8.3 \mathrm{~Hz}, \mathrm{H}-9), 8.65$ (d, $1 \mathrm{H}, J=8.4 \mathrm{~Hz}, \mathrm{H}-6)$. IR (KBr) cm $\mathrm{cm}^{-1}: 1130,1227$ (C-O-C), $1623(\mathrm{C}=\mathrm{N})$. MS $(\mathrm{m} / \mathrm{z}): 356(\mathrm{M}+1)$. Anal. Calcd. for $\mathrm{C} 16 \mathrm{H} 11$ BrN4O: C, 54.10; H, 3.12; N, 15.77. Found: C,54.23; H, 3.28; N, 15.56 .

5-(4-Methylbenzyloxy)tetrazolo[1,5-a]quinoline (4s): $\mathrm{mp}$ $240 \sim 242{ }^{\circ} \mathrm{C}$; yield 76.9\%. ${ }^{1} \mathrm{H}-\mathrm{NMR}\left(\mathrm{CDCl}_{3}, 300 \mathrm{MHz}\right): \delta 5.33$ $\left(\mathrm{s}, 2 \mathrm{H}, \mathrm{OCH}_{2}\right), 7.10(\mathrm{~s}, 1 \mathrm{H}, \mathrm{CH}=), 7.26(\mathrm{~d}, 2 \mathrm{H}, J=7.9 \mathrm{~Hz}, \mathrm{Ar}-\mathrm{H})$, 7.41 (d, 2H, J=7.9 Hz, Ar-H), 7.68 (t, 1H, J=7.7 Hz, H-7), 7.87 (t, 1H, $J=7.8 \mathrm{~Hz}, \mathrm{H}-8), 8.33$ (d, 1H, $J=8.1 \mathrm{~Hz}, \mathrm{H}-9), 8.63$ (d, $1 \mathrm{H}, J=8.3 \mathrm{~Hz}, \mathrm{H}-6)$. IR (KBr) cm ${ }^{-1}: 1126,1225$ (C-O-C), $1620(\mathrm{C}=\mathrm{N})$. $\mathrm{MS}(\mathrm{m} / \mathrm{z}): 291(\mathrm{M}+1)$. Anal. Calcd. for $\mathrm{C} 17 \mathrm{H} 14$ N4O: C, 70.33; H, 4.86; N, 19.30. Found: C, 7.48; H, 3.94; N, 19.11 .

Pharmacology. The anticonvulsant evaluation was undertaken following the protocols of the phase I tests of the ADD (Antiepileptic Drug Development) program. ${ }^{11,12}$ Antidepressant activity of the derivatives was measured by FST. ${ }^{13}$ The tested compounds were suspended in aqueous Tween $80(3 \% \mathrm{v} / \mathrm{v}, 0.9 \%$ $\mathrm{NaCl})$. KunMing mice ( $22 \pm 2 \mathrm{~g}$ ) were used for all pharmacology experiments. Mice were housed collectively in groups of ten in polycarbonate cages. They were maintained on a $12 \mathrm{~h}$ light/dark cycle in a temperature controlled $\left(23 \pm 2{ }^{\circ} \mathrm{C}\right)$ laboratory. Food and water were available ad libitum.

Anticonvulsant activity: All compounds were administered intraperitoneally (i.p.) at a volume of $0.005 \mathrm{~mL} / \mathrm{g}$ body weight for mice at doses of 30,100 , or $300 \mathrm{mg} / \mathrm{kg}$ to three animals. Activity was evaluated using the MES test. The rotarod test was used to investigate the neurotoxicity of the compounds.

Maximal electroshock seizure (MES) test - Seizures were elicited with a $60 \mathrm{~Hz}$ alternating current of $50 \mathrm{~mA}$ intensity in mice. The current was applied via corneal electrodes for $0.2 \mathrm{~s}$. Protection against the spread of MES-induced seizures was defined as the abolition of the hind-leg tonic extension component of the seizure. 
Neurotoxicity - The rotarod test was used to evaluate neurotoxicity. The animal was placed on a 1 inch diameter wooden rod rotating at $6 \mathrm{rpm}$. Normal mice remain on the rod at this speed. Failure of the animal to remain on the rod for 1 min was taken to be a sign of neurologic toxicity.

Antidepressant activity: All compounds were administered intraperitoneally at a volume of $0.005 \mathrm{~mL} / \mathrm{g}$ body weight for male mice at doses of $100 \mathrm{mg} / \mathrm{kg}$ to six animals. Antidepressant activity was evaluated using the FST test.

Forced swimming test(FST) - Male KunMing mice (20 - 24 g) were used in the forced swimming test. ${ }^{13}$ On testing day, mice were assigned to different groups ( $\mathrm{n}=6$ for each group). The synthesized compounds and the standard drug Fluoxetine were given as an intraperitoneal injection to mice. Control animals received $3 \%$ aqueous solution of Tween 80 . Thirty minutes later, the mice were dropped one at a time into a Plexiglas cylinder ( $25 \mathrm{~cm}$ height, diameter $10 \mathrm{~cm}$ containing water to a height of $10 \mathrm{~cm}$ at $23 \sim 25^{\circ} \mathrm{C}$ ) and observed for $6 \mathrm{~min}$. After the first $2 \mathrm{~min}$ of the initial vigorous struggling, the animals were immobile. A mouse was judged immobile if it floated in the water in an upright position and made only slight movements to prevent sinking. The total duration of immobility was recorded during the last 4 min of the 6 min test.

5-Hydroxytryptophan (5-HTP) induced mouse head-twitch test - To investigate whether the serotonergic system was involved in the antidepressant-like effect of compound $\mathbf{4 k}$, we performed a 5-HTP induced head-twitch test. ${ }^{18,19}$ Mice were administered intraperitoneally with compound $\mathbf{4 k}(100 \mathrm{mg} / \mathrm{kg})$, Fluoxetine (FLU) $(30 \mathrm{mg} / \mathrm{kg})$ or $3 \%$ aqueous solution of Tween $8030 \mathrm{~min}$ before DL-5-HTP (300 mg/ $\mathrm{kg}$, i.p.). Immediately after the second injection, mice were placed into plastic cages.
Ten minutes later, the cumulative number of head twitches (rapid movements of the head with little or no involvement of the trunk) was recorded for $6 \mathrm{~min}$.

Yohimbine toxicity potentiation test-To reveal whether the noradrenergic system is involved in the antidepressant-like effect of the compound $\mathbf{4 k}$, the yohimbine toxicity potentiation test was performed. ${ }^{18,19}$ Mice were treated with compound $\mathbf{4 k}(30$, 50 and $100 \mathrm{mg} / \mathrm{kg}$ ), clomipramine $(30 \mathrm{mg} / \mathrm{kg})$ or $3 \%$ aqueous solution of Tween 80 intraperitoneal injection $0.5 \mathrm{~h}$ prior to yohimbine administration ( $25 \mathrm{mg} / \mathrm{kg}$, subcutaneous injection). The number of dead mice was calculated during a $20 \mathrm{~h}$ period after the injection of yohimbine.

\section{Results and Discussion}

As shown in Scheme 1, a series of 5-alkoxy-tetrazolo[1,5-a] quinolines were synthesized. The structures of new compounds were characterized by spectral methods and elementary analysis. All spectral data corroborated the assumed structures.

Anticonvulsant activities of the synthesized compounds were assessed by maximal electroshock (MES) as shown in Table 1. According to the results of the experiments, it is clear that anticonvulsant activities of all compounds were weak. Only 5-methoxytetrazolo[1,5-a]quinoline (4a), 5-ethoxytetrazolo[1, 5-a]quinoline (4b) and 5-propoxytetrazolo[1,5-a]quinoline (4c) exhibited activity against MES induced seizures at $300 \mathrm{mg} / \mathrm{kg}$ dose level. Neurotoxicity was not observed in any of the synthesized compounds at a dose of $300 \mathrm{mg} / \mathrm{kg}$ (Table 1).

Antidepressant activities of the compounds were investigated with the forced swimming test (FST). FST, a behavioral test, is commonly used to predict the activity of antidepressants. ${ }^{13}$

Table 1. Phase I anticonvulsant screening of the compounds

\begin{tabular}{|c|c|c|c|c|c|c|c|c|c|c|c|c|}
\hline \multirow{3}{*}{ Compounds } & \multicolumn{6}{|c|}{$\operatorname{MES}^{a}$} & \multicolumn{6}{|c|}{ Toxicity $^{b}$} \\
\hline & \multicolumn{3}{|c|}{$1 / 2 \mathrm{~h}$} & \multicolumn{3}{|c|}{$4 \mathrm{~h}$} & \multicolumn{3}{|c|}{$1 / 2 \mathrm{~h}$} & \multicolumn{3}{|c|}{$4 \mathrm{~h}$} \\
\hline & 30 & 100 & 300 & 30 & 100 & 300 & 30 & 100 & 300 & 30 & 100 & 300 \\
\hline $4 a$ & $0 / 3$ & $0 / 3$ & $2 / 3$ & $0 / 3$ & $0 / 3$ & $0 / 3$ & $0 / 3$ & $0 / 3$ & $0 / 3$ & $0 / 3$ & $0 / 3$ & $0 / 3$ \\
\hline $4 b$ & $0 / 3$ & $0 / 3$ & $3 / 3$ & $0 / 3$ & $0 / 3$ & $0 / 3$ & $0 / 3$ & $0 / 3$ & $0 / 3$ & $0 / 3$ & $0 / 3$ & $0 / 3$ \\
\hline $4 c$ & $0 / 3$ & $0 / 3$ & $2 / 3$ & $0 / 3$ & $0 / 3$ & $0 / 3$ & $0 / 3$ & $0 / 3$ & $0 / 3$ & $0 / 3$ & $0 / 3$ & $0 / 3$ \\
\hline $4 d$ & $0 / 3$ & $0 / 3$ & $0 / 3$ & $0 / 3$ & $0 / 3$ & $0 / 3$ & $0 / 3$ & $0 / 3$ & $0 / 3$ & $0 / 3$ & $0 / 3$ & $0 / 3$ \\
\hline $4 e$ & $0 / 3$ & $0 / 3$ & $0 / 3$ & $0 / 3$ & $0 / 3$ & $0 / 3$ & $0 / 3$ & $0 / 3$ & $0 / 3$ & $0 / 3$ & $0 / 3$ & $0 / 3$ \\
\hline $4 f$ & $0 / 3$ & $0 / 3$ & $0 / 3$ & $0 / 3$ & $0 / 3$ & $0 / 3$ & $0 / 3$ & $0 / 3$ & $0 / 3$ & $0 / 3$ & $0 / 3$ & $0 / 3$ \\
\hline $4 g$ & $0 / 3$ & $0 / 3$ & $0 / 3$ & $0 / 3$ & $0 / 3$ & $0 / 3$ & $0 / 3$ & $0 / 3$ & $0 / 3$ & $0 / 3$ & $0 / 3$ & $0 / 3$ \\
\hline $4 h$ & $0 / 3$ & $0 / 3$ & $0 / 3$ & $0 / 3$ & $0 / 3$ & $0 / 3$ & $0 / 3$ & $0 / 3$ & $0 / 3$ & $0 / 3$ & $0 / 3$ & $0 / 3$ \\
\hline $4 i$ & $0 / 3$ & $0 / 3$ & $0 / 3$ & $0 / 3$ & $0 / 3$ & $0 / 3$ & $0 / 3$ & $0 / 3$ & $0 / 3$ & $0 / 3$ & $0 / 3$ & $0 / 3$ \\
\hline $4 j$ & $0 / 3$ & $0 / 3$ & $0 / 3$ & $0 / 3$ & $0 / 3$ & $0 / 3$ & $0 / 3$ & $0 / 3$ & $0 / 3$ & $0 / 3$ & $0 / 3$ & $0 / 3$ \\
\hline $4 k$ & $0 / 3$ & $0 / 3$ & $0 / 3$ & $0 / 3$ & $0 / 3$ & $0 / 3$ & $0 / 3$ & $0 / 3$ & $0 / 3$ & $0 / 3$ & $0 / 3$ & $0 / 3$ \\
\hline 41 & $0 / 3$ & $0 / 3$ & $0 / 3$ & $0 / 3$ & $0 / 3$ & $0 / 3$ & $0 / 3$ & $0 / 3$ & $0 / 3$ & $0 / 3$ & $0 / 3$ & $0 / 3$ \\
\hline $4 m$ & $0 / 3$ & $0 / 3$ & $0 / 3$ & $0 / 3$ & $0 / 3$ & $0 / 3$ & $0 / 3$ & $0 / 3$ & $0 / 3$ & $0 / 3$ & $0 / 3$ & $0 / 3$ \\
\hline $4 n$ & $0 / 3$ & $0 / 3$ & $0 / 3$ & $0 / 3$ & $0 / 3$ & $0 / 3$ & $0 / 3$ & $0 / 3$ & $0 / 3$ & $0 / 3$ & $0 / 3$ & $0 / 3$ \\
\hline 40 & $0 / 3$ & $0 / 3$ & $0 / 3$ & $0 / 3$ & $0 / 3$ & $0 / 3$ & $0 / 3$ & $0 / 3$ & $0 / 3$ & $0 / 3$ & $0 / 3$ & $0 / 3$ \\
\hline $4 p$ & $0 / 3$ & $0 / 3$ & $0 / 3$ & $0 / 3$ & $0 / 3$ & $0 / 3$ & $0 / 3$ & $0 / 3$ & $0 / 3$ & $0 / 3$ & $0 / 3$ & $0 / 3$ \\
\hline $4 q$ & $0 / 3$ & $0 / 3$ & $0 / 3$ & $0 / 3$ & $0 / 3$ & $0 / 3$ & $0 / 3$ & $0 / 3$ & $0 / 3$ & $0 / 3$ & $0 / 3$ & $0 / 3$ \\
\hline $4 r$ & $0 / 3$ & $0 / 3$ & $0 / 3$ & $0 / 3$ & $0 / 3$ & $0 / 3$ & $0 / 3$ & $0 / 3$ & $0 / 3$ & $0 / 3$ & $0 / 3$ & $0 / 3$ \\
\hline $4 s$ & $0 / 3$ & $0 / 3$ & $0 / 3$ & $0 / 3$ & $0 / 3$ & $0 / 3$ & $0 / 3$ & $0 / 3$ & $0 / 3$ & $0 / 3$ & $0 / 3$ & $0 / 3$ \\
\hline
\end{tabular}

${ }^{a}$ Maximal electroshock test (number of animals protected/number of animals tested). ${ }^{b}$ Rotarod toxicity (number of animals exhibiting toxicity/number of animals tested). 
Table 2. Antidepressant activities of the compounds evaluated by FST

\begin{tabular}{cccc}
\hline Compounds & $\begin{array}{c}\text { Dose } \\
(\mathrm{mg} / \mathrm{kg})\end{array}$ & $\begin{array}{c}\text { Duration of } \\
\text { immobility (s) }\end{array}$ & $\begin{array}{c}\text { Change from } \\
\text { control (\%) }\end{array}$ \\
\hline $\mathbf{4 a}$ & 100 & $138.5 \pm 13.2^{a}$ & -13.87 \\
$\mathbf{4 b}$ & 100 & $105.8 \pm 14.6^{a}$ & -34.20 \\
$\mathbf{4 c}$ & 100 & $141.7 \pm 20.2$ & -11.88 \\
$\mathbf{4 d}$ & 100 & $145.8 \pm 23.3$ & -9.33 \\
$\mathbf{4 e}$ & 100 & $115.3 \pm 16.4^{a}$ & -28.30 \\
$\mathbf{4 f}$ & 100 & $155.8 \pm 15.0$ & -3.11 \\
$\mathbf{4 g}$ & 100 & $41.7 \pm 11.7^{a}$ & -74.07 \\
$\mathbf{4 h}$ & 100 & $91.7 \pm 19.7^{a}$ & -42.97 \\
$\mathbf{4 i}$ & 100 & $34.2 \pm 4.9^{a}$ & -78.73 \\
$\mathbf{4 j}$ & 100 & $57.5 \pm 14.1^{a}$ & -64.24 \\
$\mathbf{4 k}$ & 100 & $24.2 \pm 7.4^{a}$ & -84.95 \\
& 30 & $52.3 \pm 16.6^{a}$ & -66.85 \\
$\mathbf{4 l}$ & 100 & $85.5 \pm 12.5^{a}$ & -46.83 \\
$\mathbf{4 m}$ & 100 & $120.2 \pm 14.2^{a}$ & -25.25 \\
$\mathbf{4 n}$ & 100 & $99.7 \pm 12.8^{a}$ & -38.00 \\
$\mathbf{4 0}$ & 100 & $95.2 \pm 28.3^{a}$ & -40.80 \\
$\mathbf{4 p}$ & 100 & $149.7 \pm 17.9$ & -6.90 \\
$\mathbf{4 q}$ & 100 & $158.3 \pm 16.4$ & -1.55 \\
$\mathbf{4 r}$ & 100 & $155.8 \pm 13.4$ & -3.11 \\
$\mathbf{4 s}$ & 100 & $153.8 \pm 19.2$ & -4.35 \\
Fluoxetine & 30 & $76.7 \pm 13.9^{a}$ & -52.30 \\
Control & $5 \mathrm{~mL} / \mathrm{kg}$ & $160.8 \pm 4.6$ & - \\
\hline
\end{tabular}

Values represent the mean \pm S.E.M. $(n=6) .{ }^{a}$ Significantly compared to control (student-t test; $p<0.01$ ).

Table 3. Effect of $4 \mathrm{k}(30,50$ and $100 \mathrm{mg} / \mathrm{kg}$, i.p.) and Clomipramine $(30 \mathrm{mg} / \mathrm{kg}$, i.p.) on yohimbine-induced lethality in mice

\begin{tabular}{ccccc}
\hline \multirow{2}{*}{ Group } & \multirow{2}{*}{$\begin{array}{c}\text { Dose } \\
(\mathrm{mg} / \mathrm{kg})\end{array}$} & $\begin{array}{c}\text { Yohimbine } \\
(\mathrm{mg} / \mathrm{kg})\end{array}$ & \multicolumn{2}{c}{ Lethality } \\
\cline { 4 - 5 } & & 25 & 8 & 1 \\
\hline Control & & 25 & 8 & $6^{a}$ \\
Clomipramine & 30 & 25 & 8 & 0 \\
$\mathbf{4 k}$ & 30 & 25 & 8 & 1 \\
$\mathbf{4 k}$ & 50 & 25 & 8 & 1 \\
$\mathbf{4 k}$ & 100 & 25 & & \\
\hline
\end{tabular}

$n=8 .{ }^{a} p<0.05$ compared with control (Fisher's exact test).

This method may also predict the antidepressant potency in humans. ${ }^{20}$ The obtained data on the antidepressant activities of the compounds and fluoxetine are given in Table 2. In this study, all of the compounds except 4c, 4d, 4f, 4p, 4q, 4r and 4s significantly reduced the duration of immobility times at 100 $\mathrm{mg} / \mathrm{kg}$ compared to control $(\mathrm{p}<0.01)$. Among all synthesized compounds, 5-(2-fluorobenzyloxy)tetrazolo[1,5-a]quinoline (4k) was the most promising compound and significantly reduced the duration of immobility time by $66.85 \%$ at a dose of $30 \mathrm{mg} / \mathrm{kg}$ compared with the control $(\mathrm{p}<0.01)$.

Analyzing the antidepressant activities of synthesized compounds $\mathbf{4 a - 4 s}$, the following structure activity relationship was gained. From compounds $\mathbf{4 a - 4 i}$, as the length of the alkyl chain increased from one to 12 , alternating variations of activity were observed. Compounds $\mathbf{4 j - 4 s}$ were substituted by benzyl groups. Compared with compound $\mathbf{4} \mathbf{j}$, each compound with an $\mathrm{F}$ group

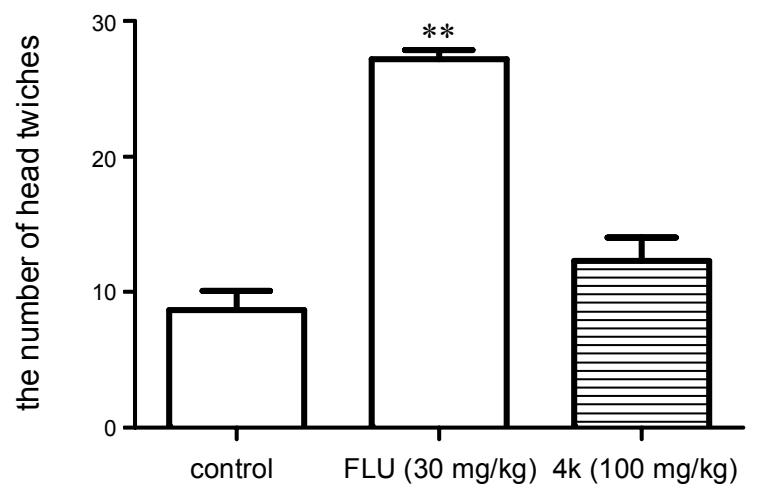

Figure 1. Effect of $4 \mathrm{k}(100 \mathrm{mg} / \mathrm{kg}$, i.p.) or fluoxetine (FLU, $30 \mathrm{mg} / \mathrm{kg}$, i.p.) on number of 5-HTP-induced head twitches in mice. Each column represents the mean \pm S.E.M., $n=10 .{ }^{*} p<0.01$, compared with control (Student t-test).

on the benzyl ring, except $p-\mathrm{F}$, exhibited enhanced antidepressant activity. In contrast, compounds carrying $p-\mathrm{Cl}$ or $p-\mathrm{Br}$ on the benzyl ring showed lower activity. Comparisons of the halogen substituted derivatives indicated that different halides contributed to the anticonvulsant activity with a rank order of $\mathrm{F}>$ $\mathrm{Cl}>\mathrm{Br}$. Compared to the derivatives with different F-substituted positions on the benzyl ring, their rank of activity order was $o-\mathrm{F}>m-\mathrm{F}>p$-F. Rank of activity order of the $\mathrm{Cl}$ substituted derivatives was $o-\mathrm{Cl}=m-\mathrm{Cl}>>p-\mathrm{Cl}$.

To investigate possible serotonergic involvement in the antidepressant-like effect of the compound $\mathbf{4 k}$, the 5-HTP induced head-twitch test was performed as shown in Figure 1. Pretreatment with FLU $(30 \mathrm{mg} / \mathrm{kg})$ significantly increased the cumulative number of head twitches, whereas pretreatment with $\mathbf{4 k}(100 \mathrm{mg} / \mathrm{kg})$ had little such effect. The results may indicate that there was little involvement of the serotonergic system in the antidepressant-like effects of compound $\mathbf{4 k}$.

Yohimbine can produce excessive noradrenaline release because it acts as an antagonist on presynaptic $\alpha$-adrenoceptors and may be used to evaluate the noradrenergic effects of antidepressants. $^{21}$ To reveal whether the antidepressant-like effect of the compound $\mathbf{4 k}$ is related to the noradrenergic system, the yohimbine toxicity potentiation test was performed and as shown in Table 3. Compound $\mathbf{4 k}$ does not increase mouse mortality induced by yohimbine at doses of 30,50 , or $100 \mathrm{mg} /$ $\mathrm{kg}$, indicating that the noradrenergic system may not be involved in the antidepressant-like effect of compound $\mathbf{4 k}$.

\section{Conclusion}

A series of 5-alkoxy-tetrazolo[1,5-a]quinolines were synthesized, and their spectral data corresponded with the assumed structures. Only three of the synthesized compounds (4a, $\mathbf{4 b}$, 4c) have shown anticonvulsant activity, however, the synthesized compounds have possessed remarkable antidepressant activity in general. Therefore, they may be really promising compounds for the treatment of depression. In fact, 5-(2-fluorobenzyloxy)tetrazolo[1,5-a]quinoline (4k) was the most promising compound and reduced immobility time by $66.85 \%$ at 30 $\mathrm{mg} / \mathrm{kg}$. Efforts to explore the possible antidepressant mecha- 
nism of the compound $\mathbf{4 k}$ indicated that the noradrenergic system seems not to be involved in the antidepressant-like effect of compound $4 \mathbf{k}$ and the serotonergic system seems a little to be involved. Further studies should be initiated to reveal the mechanism of the antidepressant-like effect of the compound $\mathbf{4 k}$.

Acknowledgments. This work was supported by the National Natural Science Foundation of China (No. 30760290 and No. 30860340).

\section{References}

1. Joseph, B.; Darro, F.; Behard, A.; Lesur, B.; Collignon, F.; Decaestecker, C.; Frydman, A.; Guillaumet, G.; Kiss, R. J. Med. Chem. 2002, 45, 2543-2555.

2. Xiao, Z.; Waters, N. C.; Woodard, C. L.; Li, Z.; Li, P. K. Bioorg. Med. Chem. Lett. 2001, 11, 2875-2878.

3. Nishi, T.; Kimura, Y.; Nakagawa, K. Zasshi. yaqugaku. 2000, 120, 1247-1260.

4. Oshiro, Y.; Sakurai, Y.; Sato, S.; Kurahashi, N.; Tanaka, T.; Kikuchi, T.; Tottori, K.; Uwahodo, Y.; Miwa, T.; Nishi, T. J. Med. Chem. 2000, 43, 177-189.

5. Otsubo, K.; Morita, Uchida, S. M.; Yamasaki, K.; Kanbe, T.; Shimizu, T. Chem. Pharm. Bull. 1991, 39, 2906-2909.

6. Banno, K.; Fujioka, T.; Kikuchi, T.; Oshiro, Y.; Hiyama, T.; Nakagawa, K. Chem. Pharm. Bull. 1988, 36, 4377-4388.

7. Bell, A. S.; Campbell, S. F.; Roberts, D. A.; Ruddock, K. S. J.
Med. Chem. 1989, 32, 2042-2049.

8. Quan, Z. S.; Wang, J. M.; Rho, J. R.; Kwak, K. C.; Kang, H. C.; Qun, C. S.; Chai, K. Y. Bull. Korean Chem. Soc. 2005, 26, 17571759

9. Cui, L. J.; Xie, Z. F.; Piao, H. R.; Li, G.; Chai, K. Y.; Quan, Z. S. Biol. Pharm. Bull. 2005, 28, 1216-20.

10. Xie, Z. F.; Chai, K. Y.; Piao, H. R.; Kwak. K. C.; Quan, Z. S. Bioorg. Med. Chem. Lett. 2005, 15, 4803-5.

11. Krall, R. L.; Penry, J. K.; White, B. G.; Kupferberg, H. J.; Swinyard, E. A. Epilepsia 1978, 19, 409-412.

12. Porter, R. J.; Cereghino, J. J.; Gladding, G. D.; Hessie, B. J.; Kupferberg, H. J.; Scoville, B.; White, B. G. Cleve Clin Q. 1984, 51,293-295.

13. Porsolt, R. D.; Bertin, A.; Jalfre, M. Arch. Int. Pharmacodyn. Ther. 1977, 229, 327-336.

14. Elhwuegi, A. S. Prog. Neuropsychopharmacol. Biol. Psychiatry 2004, 28, 435-51.

15. Millan, M. J. Eur. J. Pharmacol. 2004, 500, 371-84.

16. Chen, Y. L.; Hung, H. M.; Lu, C. M.; Li, K. C.; Tzeng, C. C. Bioorg. Med. Chem. 2004, 12, 6539-6546.

17. Bekhit, A. A.; El-Sayed, O. A.; Aboulmagd, E.; Park, J. Y. Eur. J. Med. Chem. 2004, 39, 249-55.

18. Voger, H. G.; Voger, W. H. Drug Discovery and Evaluation-Pharmacological Assays; antidepressant activity; Springer-Verlag: Berlin heidelberg, 1997; p 411.

19. Hanna, M. M. Bioorg. Med. Chem. 2007, 15, 7765-7772.

20. Willner, P.; Mitchell, P. J. Behav. Pharmacol. 2002, 13, 169-188.

21. Lapin, I. P. Psychopharmacology 1980, 70, 179-85. 